\title{
LETTER
}

doi:101017/\$1041610214001008

\section{Challenges in disseminating the findings of psychosocial research conducted in a non-English speaking country}

At the recent, 16th Congress of the International Psychogeriatric Association, the Psychosocial Research Consortium to Advance the Mental Health of Older People in the Asia-Pacific region (PROMOTE) was launched by IPA president Henry Brodaty in part to enable collaboration and dissemination of findings in this region. At the symposium that inaugurated PROMOTE, Professor Brodaty stated, "As local (psychosocial) research, conducted in many of the countries in the region, is often published in their own language, little is known about their research outside their countries." (Brodaty, 2013).

At the symposium, I discussed the two main challenges in disseminating findings of psychosocial research conducted in a non-English speaking country.

The first challenge occurs where a research project uses scales developed in the local language as measurement tools. As the supplemental figure (see Figure S1, available as supplementary material attached to the electronic version of this paper at www.journals.cambridge.org/jid_IPG) indicates, when conducting psychosocial research, the standard is for researchers to explore whether an appropriate assessment is available, and in which language.

The right stem of the supplemental flowchart shows that if such a scale is available only in the local language, the research can be conducted locally without difficulty. In general, such scales are often already validated in the local language and their employment allows researchers to capture local cultural distinctions or connotations, or simply the acceptability of the questions and the format, allowing a more expeditious response to urgent issues in the researcher's own country. For example, researchers can conduct their study immediately, write a research report in their language, and make presentations at conferences in their own country. There is no obstacle to domestic dissemination. These are all advantages to using scales validated in the local language.
However, researchers who share their findings with international colleagues by publishing in English-language journals often encounter difficulties. In my own case (see below), reviewers commented that "such domestic scales are not internationally recognized as valid because these scales were validated only in Japanese." For some reviewers and editors, if the scale was not validated in English, the validation is not sufficient for full consideration. This is one of the disadvantages of using scales validated in the local language.

Possible measures to overcome this first challenge could be: (i) to submit to a local-language journal that publishes English-language abstracts, and (ii) to present one's work at an international conference. These measures will allow at least a summary of one's work to become available in English, which is more likely to enable international dissemination.

The second challenge for researchers in nonEnglish speaking countries is to share findings with colleagues locally and domestically in the case of a research project that uses a scale originally developed in English as the measurement tool.

The left stem of the supplementary flowchart shows that for scales originally developed in English, non-native speakers of English must go through several steps including checking the cultural equivalence of the scale, validation in the local language, and publication of such validation processes before they can start conducting research. This is time and resource consuming.

In my own case, as I was unable to find a relevant scale for measuring caregiver burden in Japan in the late 1990s, I decided to develop the Japanese version of the Zarit Burden Interview (J-ZBI). The ZBI, which assesses the extent of feelings of burden among family caregivers, has been a widely used scale in English-speaking countries (Zarit et al., 1980). We checked the cultural equivalence of the ZBI, translated and back-translated the scale, and statistically confirmed the validity of the Japanese version of the ZBI. We published this validation process in English-language journals with a view to sharing our findings internationally (Arai et al., 1997; Arai and Washio, 1999).

However, using scales validated in English causes difficulties with domestic dissemination. For the first two to three years after publishing on the validation of the J-ZBI, I encountered several 
situations, e.g. local conferences, where I realized that my work was not recognized at all in my own country, possibly because the validation of the J-ZBI was published in English rather than Japanese-language journals. This lack of recognition may have occurred because some colleagues did not know about my specific work while others who knew about it chose not to acknowledge this work because of its being published in English. Publication in English-language journals, which is strongly encouraged in many non-English-speaking countries, can prevent researchers from sharing their latest findings with local colleagues.

One of the advantages of using scales validated in English is that such scales will allow non-Englishspeaking researchers to share research findings with their international colleagues more easily. One of the disadvantages is that findings based on such scales are often not disseminated to clinicians or to academics in one's own country, as they primarily read local language journals rather than international journals published in English.

Possible measures to overcome this challenge could be: (i) to present one's work at local meetings and conferences in the local language, and (ii) to publish further findings in a local journal that has an English-language abstract. In my own case, despite a long delay in local dissemination of the J-ZBI, the above measures have made the J-ZBI and its short version a widely used and cited scale for assessing caregiver burden in Japan.

Authors from non-English-speaking backgrounds would be greatly helped to meet both challenges if English-language journals provided the option of publishing abstracts in the authors' native language, and if local language journals routinely published abstracts in English.
Compared to biomedical research, psychosocial research is more influenced by the local language and culture of the participants and researchers who are involved. Because this is the nature of psychosocial research, I strongly believe that the first step for advancing psychosocial research in the AsiaPacific region is for us to be aware of the challenges derived from the differences among local languages and cultures. To meet these challenges, we must explore ways to disseminate research findings that address the needs of this region with a rapidly expanding older population.

\section{Conflict of interest}

None.

\section{References}

Arai, Y. et al. (1997). Reliability and validity of the Japanese version of the Zarit Caregiver Burden Interview. Psychiatry and Clinical Neurosciences, 51, 281-287.

Arai, Y. and Washio, M. (1999). Burden felt by family caring for the elderly members needing care in southern Japan. Aging and Mental Health, 3, 158-164.

Brodaty, H. (2013). Psychosocial Research Consortium to Advance the Mental Health of Older People in the Asia Pacific region (PROMOTE). International Psychogeriatrics, 25, S1-S10.

Zarit, S. H. et al. (1980). Relatives of the impaired elderly: correlates of feelings of burden. Gerontologist, 20, 649-655.

\section{YUMIKO ARAI}

Department of Gerontological Policy, National Center for Geriatrics and Gerontology (NCGG), Obu, Aichi, Japan

E-mail: yarai903@ncgg.go.jp 